\title{
Microfluidics for IVD: In Pursuit of the Holy Grail \\ Peng LI*
}

Rheonix, Inc., 22 Thornwood Dr., Ithaca, NY 14850, USA

\section{Introduction}

After climbing up to the peak of inflated expectations and falling down to the trough of disillusionment, the microfluidics community has come to realize that microfluidics will neither miniaturize all the routine laboratory tests nor run through all applications with no major outlet. It will instead serve in combination with associated technologies to provide the means to generate significant leaps in performance and capabilities for the consumers as an enabling technology [1]. In the sense of an enabling technology, we have seen the successful applications of microfluidics in a variety of commercial products. Caliper's LabChip system employs a glass microfluidic chip to achieve rapid, high throughput fluorescent, electrophoretic analysis of fragments of nucleic acids and proteins. Quanta Life uses dropletbased microfluidic technologies to perform digital PCR for nucleic acid quantitation. Fluidigm's highly integrated fluidic circuits offer a low cost and high-sample throughput solution for multiplex SNP (single-nucleotide polymorphism) genotyping. Despite these excellent achievements, the impact of applying microfluidics technologies to the in vitro diagnostics (IVD) industry to fully automate the sophisticated molecular diagnostics process will be far more profound.

Since its inception, microfluidics has shown a tremendous potential to become an important tool for IVD. "The Holy Grail" of microfluidics-based IVD is to offer a hand-held/portable, standalone, self-calibrating, automated, and inexpensive device that is capable of performing rapid, specific, sensitive, and quantitative tests for multiple analytes using minimal raw samples. However, so far, only very limited microfluidic devices have been sparsely used for such applications. Why this long advertised promise has yet to be delivered?

\section{IVD market}

The in vitro diagnostics market, while appealing, is very difficult to penetrate. First, although IVD provides critical health information for patient outcome, its cost accounts for only a tiny fraction of overall healthcare spending. Second, IVD is a very competitive and also rapidly evolving market. In this environment, established technologies will often set the standards in terms of technical performance and cost that any new device will have to beat. Third, the IVD market is under the regulation and supervision of agencies such as the U.S. Food and Drug Administration (FDA). The manufacturer must go through appropriate application processes in order to obtain FDA clearance/approval for marketing. Fourth, the market acceptance of a product will be largely affected by the availability of coverage and reimbursement from health insurance providers.

\section{Micro-plumbing clinical samples}

Although many intricate micro pumping, valving, and mixing technologies have been invented in academic laboratories around the world, very limited of them have proven to handle clinical samples reliably. Microfluidic devices that are developed and validated only with degased and filtered laboratory buffers would most likely fail with clinical samples, such as whole blood, vaginal swabs, biopsy specimens, saliva, etc., because these real-world samples are often viscous, prone to bubble formation, and contain particles that can clog microchannels.
In addition, for many applications, the volume of the sample needs to be large enough to have clinical relevance. The required sample volume (e.g. $1 \mathrm{ml}$ ) can often be thousands of times higher than the volume of a microreactor (e.g. $1 \mu \mathrm{L}$ ) on a microfluidic device. For example, the sensitivity requirement of encephalitis test may be as low as 1 to 100 $\mathrm{PFU} / \mathrm{mL}$ from cerebrospinal fluid. This means that even a microfluidic device with a high detection sensitivity still needs to process a significant volume of cerebrospinal fluid to resolve that 1 to $100 \mathrm{PFU} / \mathrm{mL}$. Another difficulty of handling clinical samples lies in the intrinsic high surfaceto-volume ratio of microfluidic channels. The high surface-to-volume ratio can cause analyte depletion and increased nonspecific adsorption.

\section{Manufacturability}

One key factor that determines the market acceptance of IVD devices is the cost per reportable result and the price of the necessary equipment base. Many microfluidic startups or pilot programs failed because people didn't stop to evaluate the cost of manufacturing their devices at the early stage of developing the technologies. If these devices cannot be manufactured with relative ease at minimum cost and maximum reliability in large scale, they will either be too expensive for the consumers or even never get out of the lab. The manufacturability of microfluidic cartridges is largely affected by the material selection and the fabrication method. For instance, can a competitive market price be achieved when the cartridges are fabricated with glass and integrated with micro-optical elements and gold electrodes? In addition, multilayer fabrication does provide sophisticated functionalities in terms of liquid handling, optics, and so forth. But if you consider a five step lamination process with $95 \%$ yield for each step, this brings down the final yield to only $77 \%$.

\section{Interfacing and integration}

The lack of a universal microfluidic interface has apparently become a hurdle to the interconnection of various commercially available microfluidic components, which makes it very difficult for one company to directly take advantage of the achievements of the others. An ideal approach would be a plug-and-play interface between various components in a microfluidic system. For example, these components can be essential fluid handling elements such as micropumps, valves, and mixers. They can also be integral functional units such as a nucleic acid purifier and a capillary electrophoresis module for analyzing purified DNA. Another issue with the interfacing is the connection of a microfluidic chip with the rest of the off-chip devices, which is often

${ }^{*}$ Corresponding author: Peng $\mathrm{Li}$, Application scientist Rheonix, Inc., 22 Thornwood Dr., Ithaca, NY 14850, USA, Tel: (607) 257-1242x183; Fax: (607) 257-0979; E-mail: pli@rheonix.com

Received January10, 2012; Accepted January 12, 2012; Published January 13 2012

Citation: Li P (2012) Microfluidics for IVD: In Pursuit of the Holy Grail. J Bioengineer \& Biomedical Sci S8:e001. doi:10.4172/2155-9538.S8-e001

Copyright: (C) $2012 \mathrm{Li} \mathrm{P}$. This is an open-access article distributed under the terms of the Creative Commons Attribution License, which permits unrestricted use, distribution, and reproduction in any medium, provided the original author and source are credited. 
known as the world-to-chip interface. Although the amount of such interfacing efforts can be decreased with the degree of integration of the chip, the sample-to-chip interface can never be eliminated. Can some of the existing standard connectors such as Luer lock be an option? Or the sample loading interface can just simply be a sample reservoir. Any type of these interfaces will need to be designed carefully to minimize dead volume and ease subsequent sample processing steps. System integration, which is also inevitably affected by the interfacing of microfluidic components, even poses a bigger challenge because it requires combination of fluidic, mechanical, electronic, and optical units into a limited space. Interfacing and integrating these functional units and dealing with the effects of possible cross-functional interactions are all parts of this challenge.

\section{Quality control and regulatory issues}

In order to get the product through the FDA approval process, quality control with these microfluidic diagnostic devices will pose challenges. The microfluidic technologies are pushing the boundaries of what we consider traditional control processes. First, the need for handling microliter liquid requires superior accuracy and reliability for instrument control. For instance, fluid delivery with $\pm 0.5 \mu \mathrm{L}$ accuracy is considered to be high for conventional fluidic control but for microfluidic devices designed to handle $1 \mu \mathrm{L}$ purified nucleic acids this variation becomes unacceptable. Second, the strategy of performing quality control on disposable microfluidic cartridges will challenge the regulatory in terms of approval. Is it sufficient to run quality control on just a subset of a lot? This issue can get more complicated with more functional units such as nucleic acid purifier and multiplexed microarray being added to the cartridge. What about the approach of building in an internal control process on the cartridge? Third, the multidisciplinary nature of the microfluidic IVD devices brings new challenges and risks for the manufacturers when it comes to define the scope and level of the device testing needed to meet the FDA's requirements for verification and validation studies.

\section{The team}

The complexity of the field requires experts with significant scientific depth in a variety of disciplines including but limited to biology, chemistry, microfluidics, optics and electronics. The technical range of the field requires experts whose capabilities span the full development process at every scale, from system design and manufacturing to quality control and product testing. In today's diagnostics industry, building and maintaining a team of people that blends such wide-ranging capabilities pose a tremendous challenge to both the management and financing of any company.

In spite of all the difficulties aforementioned, the field seems to be unwilling to postpone the challenges and intends to win the battle. It is encouraging to see several FDA approvals and acquisitions over the past year. For example, in March 2011, Wako Diagnostics received $510(\mathrm{k})$ clearance from FDA to market its microfluidicbased clinical immunoanalyzers in the United States. Micronics was acquired by Sony in September 2011. As innovations gain stream and regulatory developments keep up, microfluidics will continue to look for opportunities in some of the new and dynamic segments of the diagnostics market.

Point-of-care testing (POCT) is currently present in the ER, the ICU, and at the bedside in every hospital in the United States. The test menu ranges from pregnancy test and glucose monitoring to the measurement of creatinine and acute cardiac markers. Microfluidic devices have been long touted for their potential to become important tools for POCT but the deployment of such devices has been very limited so far. Diagnostics for All is working on using paper-based microfluidic devices for measuring liver function outside hospital and laboratory settings [2]. The device will be about the size of a stamp and will not need external power or equipment to operate. The output will be a straightforward, semi-quantitative colorimetric reading which can be readily interpreted with telemedicine networks. The liver function test is targeted to cost $\$ 0.10$ or less. Claros Diagnostics has recently reported successful simultaneous diagnosis of HIV and syphilis by using its microfluidic chips (mChip) in Rwanda [3]. The mChip faithfully replicates all steps of ELISA using only $1 \mathrm{ml}$ of unprocessed whole blood. The mChip test does not require user interpretation of the signal.

In addition to POCT, another segment of the IVD market that is rapidly gaining strength is companion diagnostics. Companion diagnostics is emerging as a key part of personalized medicine. Particularly in oncology, patients are being better treated by drugs based on the genomic data obtained via IVD tests [4]. In July 2011, FDA released its draft guidance on in vitro companion diagnostic devices, which underscores the increasing importance diagnostics will be playing in cancer therapy and drug development. This nascent but promising field should be a niche where microfluidics can find its applications. This is because there is currently very limited FDA approved companion diagnostics technologies available for new technologies to beat. Also, microfluidic products have the potential to improve the cost-effectiveness of the development and deployment of companion diagnostic technologies. The major difficulty of implementing companion diagnostics is to obtain genetic information, particularly mutant DNA, from tumor cells from the overwhelming wild-type host DNA background. To address this challenge, Rheonix, Inc. has developed the microfluidic CARD (Chemistry and Reagent Device) molecular diagnostics platform [5]. The key component of this system is a palm-sized disposable plastic microfluidic chip that integrates all necessary steps for performing sophisticated molecular diagnostics. These steps include sample processing, extraction/purification of nucleic acid, multiplex PCR amplification, and finally detection. Rheonix is currently developing a fully automated CARD assay for the detection of different KRAS mutations. KRAS is a signaling protein that is downstream from epidermal growth factor receptors (EGFR), and clinical studies have shown that EGFR-inhibiting drugs such as panitumumab (Vectibix) and cetuximab (Erbitux) are ineffective in patients with colorectal cancer who have KRAS mutations. Thus far, as little as one copy of mutant DNA over a background of 10,000 copies of wild-type DNA (i.e., $0.01 \%$ ) can be detected from fresh or FFPE (formalin-fixed paraffin-embedded) biopsy specimens. The Rheonix CARD technology is expected to enter U.S. clinical studies during early 2012 for several personalized medicine and companion diagnostic applications.

Given all the technical, regulatory, and economic constraints discussed in this article, the efforts of commercializing microfluidic IVD devices will certainly be a long and arduous process. However, I strongly believe that, in the next decade, we will start to see more commercially available integrated microfluidic systems being used in hospital laboratories, reference laboratories as well as point-of-care settings. A domain of microfluidics technologies [6] will eventually emerge as the field matures. Towards this goal, the industry, the academia, and the regulators need to work together to nurture this field and to produce high-quality diagnostic devices for the best possible patient care. 


\section{Acknowledgments} Inc.

The author is grateful for helpful discussions with Dr. Peng Zhou at Rheonix,

\section{References}

1. Mukhopadhyay $R$ (2009) Microfluidics: on the slope of enlightenment. Anal Chem 81: 4169-4173.

2. www. dfa.org

3. Chin CD, Laksanasopin T, Cheung YK, Steinmiller D, Linder V, et al. (2011)
Microfluidics-based diagnostics of infectious diseases in the developing world. Nat Med 17: 1015-1019.

4. August J (2010) Market watch: emerging companion diagnostics for cancer drugs. Nat Rev Drug Discov 9: 351.

5. Spizz G (2011) Rheonix CARD Technology: an innovative and fully automated molecular diagnostic device. The Journal of Near-Patient Testing \& Technology (in press).

6. Rotman D (2010) Shoveling Water-Why does it take so long to commercialize new technologies? Technology Review.

This article was originally published in a special issue, Microfludics handled by Editor(s). Dr. Peng li, Rheonix, Inc, USA. 